\title{
El proceso de gramaticalización del marcador epistémico deverbal sabes
}

\author{
M. ${ }^{a}$ Elena Azofra Sierra (Universidad Nacional de Educación a Distancia, UNED, España)
}

Renata Enghels (Universiteit Gent, Bélgica)

\begin{abstract}
Resumen: Este artículo tiene el objetivo de reconstruir detalladamente el proceso, los contextos y las motivaciones de gramaticalización seguidos por el marcador epistémico sabes en su evolución histórica desde su fuente verbal. Un microanálisis diacrónico que estudia de cerca el proceso de surgimiento y de fijación de sabes entre los siglos XIX y XXI permite observar una serie de cambios semánticos, funcionales, morfosintácticos que coinciden con los criterios de gramaticalización asumidos. Los datos muestran que el marcador epistémico no parece haber llegado aún a la fase final del proceso de gramaticalización, pero sí apuntan a que el proceso se ha acelerado a partir de la segunda mitad del s. XX.
\end{abstract}

\section{Introducción}

Es bien sabido que el lenguaje hablado informal se caracteriza por la alta frecuencia de elementos marcadores que estructuran el discurso añadiendo al mensaje al mismo tiempo una dimensión subjetiva (valorando su conocimiento, como en el caso del marcador creo) o intersubjetiva (invitando al oyente a participar en el acto de comunicación). Dentro de este conjunto de elementos pragmáticos variados, los marcadores deverbales han recibido una atención particular: se trata de marcadores como mira, oye (Cuenca y Marín 2000), venga y vaya (Tanghe 2013), relacionados con los verbos de percepción y de movimiento respectivamente. También constituyen una fuente privilegiada de marcadores los verbos cognitivos: así tenemos marcadores como I think (Van Bogaert 2011), creo o pienso (Posio 2014), que reciben el nombre de epistémicos y se utilizan para expresar las creencias del hablante y/o su implicación en el discurso.

Entre los marcadores epistémicos cabe agrupar el español sabes, que tiene paralelos en otras lenguas: francés tu sais (Dostie y Sève 1999) o inglés you know. Existe un número muy elevado de estudios dedicados al marcador you know en inglés, y más particularmente centrados en marco histórico que nos interesa aquí; autores como Macaulay (2002), House (2009) o Vincent et al. (2009) defienden que el marcador ha llegado al punto final de un largo proceso de gramaticalización, y que por consiguiente se define debidamente como marcador discursivo. Contrariamente, a pesar de su alta frecuencia en el discurso, el marcador sabes ${ }^{1}$ aún no ha recibido la atención que merece en la literatura sobre los marcadores discursivos en español. A primera vista, su empleo es bastante paralelo al de you know, como apreciamos en los ejemplos (1a-b).

(1) a. At at least at the very smallest scale we can do something... but you're quite right you know in larger scale you know that's true you know because eh majority of eh of eh [...]. (apud House 2009: 180)

b. [...] entonces desde la sierra en ¿sabes? andamos mogollón / no sé qué / y ya pensé / bueno / pues cuando bajemos ya la llamo //y estaban todos ahí superpendientes ¿sabes? de / ¿sabes? porque yo estaba todo el tiempo pensando / es que / estoy segura / de que Cristina va a empezar a [/] a preguntarme o / ¿sabes? [/] o [...]. (C-Oral-Rom, 2000)

Sin embargo, mientras que el estatus de you know como marcador discursivo no se discute, observamos más controversia en la literatura sobre el español sabes. Por un lado, autores como Ortega Olivares (1986), Boyero Rodríguez (2002), López Serena y Borreguero Zuloaga (2010) y

\footnotetext{
${ }^{1}$ Como veremos a lo largo de este estudio, el establecimiento de la modalidad interrogativa del marcador a veces resulta problemático, de ahí que hayamos optado por referirnos a él sin utilizar signos de interrogación ("el marcador sabes"). Por supuesto, en los ejemplos citados sí copiamos fielmente su representación en el corpus, con o sin signos de interrogación (¿sabes? o sabes).
} 
Cuenca (2013) no dudan en clasificar sabes y otros marcadores deverbales de segunda persona como marcador de discurso. Lo clasifican como "marcador conversacional" (incluido entre los que llaman la atención del interlocutor, controlan su reacción, y verifican su comprensión), "interaccionales" (que controlan la recepción del mensaje) y apéndices de justificación (con los que el hablante "justifica" su mensaje para con el interlocutor). El uso del sabes sería, según estos autores, resultado del reanálisis del verbo cognitivo como marcador epistémico, incluyendo la omisión de la completiva en que, y un traslado a una posición sintáctica más periférica. En cuanto a su semántica, Santos (2003) define sabes en su diccionario de partículas como "[p]alabra fática con que se busca la atención, participación y complicidad del interlocutor más que la averiguación de si sabe o no sabe el contenido pertinente". También Fuentes (2009), en su diccionario, lo considera "marcador interactivo de contacto con el interlocutor", con distintos valores: confirmativo, continuativo, enfatizador de la información, etc.

De otro lado, en el capítulo canónico sobre los marcadores del discurso de Zorraquino y Portolés (1999: 4143), sabes se clasifica bajo los "marcadores conversacionales", que cumplen varias funciones como las de indicar el grado de certeza con respecto al contenido del mensaje, estructurar la conversación indicando un cambio de tema o de turno de palabra, negociando relaciones de cortesía entre hablante e interlocutor, etc. (de ahí el término de enfocadores de la alteridad). Sin atribuirles el estatuto pleno de marcador de discurso, los autores sostienen que formas como ves, escucha, entiendes, fíjate y sabes solo parcialmente han sufrido un proceso de gramaticalización. Las pruebas positivas incluyen el blanqueamiento semántico y la fijación morfosintáctica. Sin embargo, variaciones colocacionales (ya sabes, tú sabes, no sabes, etc.) y modales (la modalidad afirmativa e interrogativa alterna con la modalidad exclamativa), les impiden asignarles el estatus completo de marcador de discurso. ${ }^{2}$ Más recientemente, y en la misma línea de ideas, Kluge (2011: 306) afirma que:

"el proceso de pragmaticalización de sabes se ha intensificado mucho en los últimos años y acaba de
integrarse - o actualmente está integrándose - en el elenco de los marcadores del discurso del español
peninsular."

Si es cierto que la conclusión del proceso es tan reciente, debe ser posible reconstruir el proceso evolutivo de sabes mediante datos empíricos.

Ahora bien, como se puede apreciar en (2), en español contemporáneo el mismo contenido semántico puede ser comunicado con distintas expresiones entre las que hay a veces diferencias de matiz muy pequeñas, por lo que hemos hecho extensivo el análisis a otras construcciones en que está integrada la forma sabes. Tenemos en estos ejemplos las diversas expresiones epistémicas que van a ser objeto de este estudio, que incluyen varias construcciones con núcleo verbal sabes (2a-g), así como esta forma verbal aislada y con modalidad interrogativa $(2 \mathrm{~h}-\mathrm{j})$.

(2) a. Ya sabes que te quiero.

b. Tú sabes que te quiero.

c. No sabes lo que te quiero.

d. Como sabes, te quiero.

e. Te quiero. Ya lo sabes.

f. Te quiero. Y lo sabes.

g. ¿Sabes que te quiero?

h. Yo te quiero, ¿sabes?

i. ¿Sabes? yo te quiero.

j. En fin... es difícil... ¿sabes?... pero... me gustaría... Te quiero

Los valores modales de estas oraciones contrastan con el sentido léxico pleno con que puede ser usado el verbo sabes (3a-b):

(3) a. Pues yo sé una cosa que tú no sabes, aunque quizás lo presientes, y que seguramente sabrás muy pronto. (Galdós, Fortunata, 1885-1887)

\footnotetext{
${ }^{2}$ Cabe advertir que la clasificación de Martín Zorraquino y Portolés data de finales del siglo XX.
} 
b. ¿Qué cara pones tú cuando sabes que tu mujer le gusta alguien, o que tiene un amante [...]. (CREA oral, 1996)

Así, el objetivo de este trabajo es reconstruir la evolución del marcador sabes desde su estatuto como verbo cognitivo con valor epistémico pleno hasta su función como marcador multifuncional en español contemporáneo. ${ }^{3}$ La hipótesis principal es que el uso de sabes como marcador epistémico interpersonal es el resultado de una serie de cambios formales y semántico-funcionales que tuvieron lugar en el campo léxico más amplio de las expresiones epistémicas entre finales del siglo XIX y la época actual. Más concretamente, nuestro propósito es contestar a las preguntas de investigación siguientes:

(i) ¿Cómo se describe empíricamente la evolución del uso de las expresiones epistémicas observando ejemplos consecutivos del español moderno y contemporáneo?, ¿cuál es la frecuencia de sabes y sus variantes (ya sabes, no sabes, tú sabes, etc.) en el corpus?

(ii) ¿En qué medida esta evolución confirma el estatus gramaticalizado de sabes?, ¿en qué medida sabes cumple entonces las características de los marcadores del discurso?

El presente análisis se destaca entonces de cualquier estudio anterior por la idea de que no se puede estudiar el marcador sabes de manera aislada, sin integrarlo en la red compleja de expresiones epistémicas que tiene a su disposición el hispanohablante.

A estas preguntas se añade un objetivo metodológico, a saber, delimitar el alcance de la comparabilidad entre corpus de diferente naturaleza; en efecto, un primer estudio exploratorio pone a prueba nuestra metodología de recogida de datos para realizar el estudio diacrónico de un fenómeno que se observa ante todo en el lenguaje oral (por ejemplo, Hernando Cuadrado 2015) y plantea la dificultad de manejar subcorpus de distinta naturaleza.

\section{Corpus y metodología}

\subsection{Datos}

La lista de expresiones epistémicas seleccionadas (y ejemplificadas a lo largo del artículo) incluye los elementos siguientes: sabes, como sabes, no sabes, ya sabes, (tú) sabes (tú), como tú sabes, ya sabes tú, lo sabes, sabes (una) cosa, sabes qué, no lo sabes, ya lo sabes, lo sabes tú, sabes + oración, como sabes + oración, ya sabes + oración, no sabes + oración, (tú) sabes (tú) + oración, tú no sabes + oración, ya sabes tú + oración. En la selección de casos pertinentes se excluyen los usos "plenos" de sabes (como en los ejemplos bajo (3)), pero sí están incluidos los casos semánticamente intermedios entre este uso cognitivo pleno y el uso discursivo de sabes, como en (4). Solo de esta manera será posible tener una visión más clara de la evolución semántica y morfosintáctica del campo léxico vinculado al verbo saber.

(4) ¿Sabes lo que pasó después? No te lo vas a creer.

El corpus empleado para este estudio procede de varios textos y bancos de datos que documentan (o imitan) el registro oral, y más particularmente la conversación espontánea del español peninsular (ya sea culta o no, y ya sea auténtica o recreada con fines de caracterización de personajes en una novela). La diacronía estudiada se extiende desde el s. XIX hasta la época actual, si bien dividimos el estudio en dos partes, trabajando con dos subcorpus, según el período estudiado: el primer período se extiende entre finales del siglo XIX y 1975 (CORPUS I); y el segundo está comprendido entre 1975 y la actualidad (CORPUS II). Lógicamente, los datos de ambos períodos no son

\footnotetext{
${ }^{3}$ El estudio que presentamos forma parte de un proyecto más amplio sobre el marcador conversacional sabes. En una primera fase, el análisis realizado se limita al español peninsular y excluye las posibles formas que podrían ser consideradas variantes de sabes, a saber, la forma de cortesía sabe [usted] y los plurales correspondientes sabéis y saben [ustedes]; estas formas, que presentan un grado menor de fijación y frecuencia, serán analizadas en una segunda fase.
} 
completamente de la misma naturaleza (orales, espontáneos), pero hemos aspirado al máximo a la uniformidad de los subcorpus. Estos corpus proporcionan los datos empíricos que permiten reconstruir el cambio categorial del verbo cognitivo saber en el marcador epistémico sabes.

Más concretamente, el CORPUS I abarca el último cuarto del siglo XIX y los primeros tres cuartos del siglo XX, es decir, unos cien años, que dividimos en cuatro bloques de veinticinco años, a los que llamamos 19-4, 20-1, 20-2, 20-3. Estos bloques se consideran como "microdiacronías" en términos de Pons Bordería (2014), es decir, son pequeños períodos de tiempo que pueden ser de especial relevancia en la datación de un cambio lingüístico. Como punto de partida, se han seleccionado bloques en los que se podría observar una regularidad en el curso de la evolución de un fenómeno. Los datos para este período se han extraído de un conjunto de obras contenidas en el CORDE de la RAE, detalladas más abajo; todas ellas comparten una característica: la existencia de un número importante de diálogos. Hemos comenzado por la novela realista porque en ella el escritor aspira a reflejar fielmente la realidad que tiene a su alrededor, incluidos aquellos rasgos característicos del habla de una clase social, un grupo o una zona geográfica determinada. Por ejemplo, sorprende la diferencia entre el gran número de sabes que aparece en las novelas de Galdós, ambientadas en Madrid, y la escasez en las de Valera, ambientadas en el sur de España. A pesar del volumen de palabras analizadas (en total, estas obras suman, según los datos del CORDE, casi cuatro millones de palabras), destaca que el número de expresiones epistémicas no es muy grande, y menos aún el número de sabes independiente y con modalidad interrogativa: en total se han recuperado 1269 sabes en un total de 3.891 .232 palabras, lo que proporciona una frecuencia absoluta de 0.00033 (equivalente a 3.3 sabes por cada 10.000 palabras). En la tabla 1 se muestran las obras de los distintos períodos.

\begin{tabular}{|l|l|}
\hline \multicolumn{1}{|c|}{ período } & \multicolumn{1}{|c|}{ obras analizadas } \\
\hline $19-4$ & \multicolumn{1}{|c|}{ B. Pérez Galdós, Rosalía, c. 1872; E. Pardo Bazán, La Tribuna, 1883; L. Alas } \\
Clarín, Regenta, 1884-1885; J. M. Pereda, Sotileza, 1885-1888; B. Pérez Galdós, \\
Fortunata y Jacinta, 1885-1887; E. Pardo Bazán, Insolación, 1886; B. Pérez \\
Galdós, Miau, 1888; J. Valera, Juanita la Larga, 1895.
\end{tabular}

Tabla 1. Procedencia de los datos del CORPUS I 
Para la diacronía más reciente, el CORPUS II recoge datos orales que cubren cuatro décadas, de los años 70 hasta la primera década del nuevo milenio. Los datos son de índole diversa (entrevistas, conversaciones secretas, grabaciones de radio, televisión, etc.) pero lo importante es que haya conversaciones con un mínimo de dos participantes. ${ }^{4}$ Más concretamente se trata de los bancos de datos siguientes (seleccionados a partir de Enghels, Vanderschueren y Bouzouita 2015):
(a) Corpus del Habla culta de Madrid: entrevistas dirigidas, libres y grabaciones secretas;
(b) CREA ORAL: conversaciones, grabaciones de radio, televisión y teléfono; mezcla de interlocutores conocidos y desconocidos;
(c) CORLEC: conversaciones, también mayoritariamente entre hablantes de nivel sociocultural alto;
(d) COSER: parte de entrevistas semidirigidas; hablantes de nivel sociocultural bajo;
(e) C-Oral-Rom: conversaciones dirigidas y espontaneas; la mayor parte de las veces entre conocidos y mayoritariamente de nivel culto medio-alto;
(f) PRESEEA: conversaciones semidirigidas; ídem.

Para recoger con más precisión los posibles cambios ocurridos en esta etapa reciente, establecemos microdiacronías de diez años.

\subsection{Parámetros de gramaticalización analizados}

Los criterios de análisis aplicados a los datos de cada subcorpus no son nuevos, sino que se basan en teorías anteriores de la gramaticalización (por ejemplo, Garachana 1999 y 2008, Hopper y Traugott 2003, Company 2006). No queremos plantear una discusión teórica sobre los límites del término gramaticalización, pues no es nuestro propósito revisar el concepto a la luz de los datos aportados en el estudio. Partimos de una noción de gramaticalización en sentido amplio ${ }^{5}$ y así, consideramos los siguientes criterios:

(i) La frecuencia de uso de la forma sabes (independiente y con modalidad interrogativa) ${ }^{6}$ con respecto a otras expresiones epistémicas "competidoras" (Sección 3).

(ii) La fijación formal de la expresión epistémica, que suele acompañarse de un empobrecimiento sintáctico gradual (Sección 4.1).

(iii) La ampliación del alcance y de la autonomía predicativa de la expresión epistémica, pues un elemento más gramaticalizado deja de estar integrado en la secuencia oracional, como es bien sabido (Sección 4.2).

(iv) El valor semántico y/o la función que cumple sabes en el discurso: el blanqueamiento gradual de una forma se acompaña de una extensión de las funciones pragmáticas que puede efectuar (Sección 5).

\section{Frecuencia de uso}

Es bien sabido que la frecuencia creciente de una unidad lingüística es un indicador importante de que se está produciendo un cambio lingüístico, como afirman ya Thompson y Mulac (1991: 319): "an increase in an item's text frequency is an important concomitant of its grammaticalization" [una

\footnotetext{
${ }^{4}$ La mayoría de los datos corresponden a conversaciones entre personas de clase media-alta, salvo COSER, aunque a primera vista no se comporta de manera diferente en comparación con otros corpus.

5 Siguiendo a Company (2004: 65) consideramos la gramaticalización como un término hiperónimo refiriendo a "un macrocambio dinámico, un cambio de cambios, que engloba distintas subclases y procesos [...] la rutinización o cristalización del uso, sea cual sea la direccionalidad del cambio".

${ }^{6}$ A veces, la modalidad interrogativa no está marcada gráficamente en los corpus o en las obras literarias. Sobre la dificultad de distinguir la modalidad oracional asertiva vs. interrogativa, ver la nota 9.
} 
subida de la frecuencia textual de un elemento es un concomitante importante de su gramaticalización]. En la misma línea de ideas, el aumento de la frecuencia relativa ha sido correlacionado con la rutinización de un patrón discursivo (cf. por ejemplo Bybee 2006).

Si se calcula la frecuencia relativa (sobre 10.000 palabras) de (a) el conjunto de expresiones epistémicas con sabes, enumeradas en 2.1 , y (b) el marcador sabes de forma independiente, se obtienen los datos cuantitativos reflejados en las tablas 2 y 3.

Primero, en el CORPUS I se aprecia un aumento considerable de la frecuencia de expresiones epistémicas en el último período (20-3), que comienza a mediados del siglo XX. Además de este aumento del conjunto total de expresiones, se observa también una subida de frecuencia de sabes independiente interrogativo (de 0.3 constante en los bloques cronológicos previos pasamos a una frecuencia de 0.8 ).

\begin{tabular}{|c|c|c|c|c|c|}
\hline \multicolumn{2}{|c|}{ periodos } & \multicolumn{2}{c|}{ expres. epistémicas } & \multicolumn{2}{c|}{ sabes } \\
\hline & $\#$ palabras & $\#$ & frec. relativa & $\#$ & frec. relativa \\
\hline $\mathbf{1 9 - 4}$ & 1.241 .217 & 271 & 2.2 & 33 & 0.3 \\
\hline $\mathbf{2 0 - 1}$ & 972.238 & 150 & 1.5 & 26 & 0.3 \\
\hline $\mathbf{2 0 - 2}$ & 755.100 & 135 & 1.8 & 22 & 0.3 \\
\hline $\mathbf{2 0 - 3}$ & 922.677 & 348 & 3.8 & 77 & 0.8 \\
\hline
\end{tabular}

Tabla 2. Frecuencias CORPUS I

En cuanto a los datos del CORPUS II, observamos que, si se compara la década de los años 70 con datos de los años 2000, la frecuencia relativa del uso de las expresiones epistémicas crece, aunque la diferencia es bastante limitada. ${ }^{7}$ Sin embargo, el uso de sabes aislado crece también, pero esta vez de forma más significativa: de un 1.9 en el Habla culta de Madrid (década de los 70) a una frecuencia relativa de 5.7 en el corpus $C$-Oral-Rom (> 2000).

\begin{tabular}{|l|l|l|l|c|c|c|}
\hline \multicolumn{2}{|c|}{ corpus } & década & \multicolumn{2}{c|}{ expres. epistémicas } & \multicolumn{2}{c|}{ sabes } \\
\hline & $\#$ palabras & & $\#$ & frec. relativa & $\#$ & frec. relativa \\
\hline Habla de Madrid & aprox. 150.000 & 70 & 73 & 4.8 & 28 & 1.9 \\
\hline CREA oral & aprox. 6 millones $?$ & $80-90$ & 420 & $*^{8}$ & 235 & $*$ \\
\hline CORLEC & 269.500 & $90-2000$ & 84 & 3.1 & 65 & 2.4 \\
\hline Coser & aprox. 425.000 & $90-2000$ & 168 & 4 & 103 & 2.4 \\
\hline C-Oral-Rom & 300.000 & $>2000$ & 216 & 7.2 & 172 & 5.7 \\
\hline PRESEEA & 216.046 & $>2000$ & 118 & 5.5 & 93 & 4.3 \\
\hline
\end{tabular}

Tabla 3. Frecuencias CORPUS II

\footnotetext{
${ }^{7}$ Otras variaciones en la frecuencia de uso se deben posiblemente a los datos incluidos en los corpus: por ejemplo, las conversaciones registradas en el C-Oral-Rom incluyen más datos espontáneos que las del PRESEEA, lo cual se relaciona con una frecuencia relativa respectivamente más o menos alta (de 7.2 a 5.5.) de expresiones epistémicas.

${ }^{8}$ Para CREA ORAL no conocemos el número total de palabras, por lo que resulta imposible calcular las frecuencias.
} 
Pasamos a analizar la frecuencia del conjunto de ocurrencias de sabes y de expresiones epistémicas respecto al total de palabras (gráfico 1) y la frecuencia relativa de sabes aislado respecto al número total de expresiones epistémicas más complejas (gráfico 2).

El gráfico 1 muestra la frecuencia relativa sobre 10.000 palabras en los dos subcorpus, tanto de las expresiones epistémicas (líneas superiores), como del sabes aislado (líneas inferiores). A la izquierda de la línea de puntos están las curvas del CORPUS I y a la derecha se sitúan las curvas del CORPUS II. En el CORPUS I (hasta 1975) la frecuencia de las expresiones epistémicas crece más rápidamente que el sabes aislado, que se mantiene constante aunque experimenta una subida en el último microperíodo (1951-1975). Sin embargo, es evidente que, en el CORPUS II, ya en la década de los 70 encontramos unos valores muy superiores a las del CORPUS I, lo cual se debe a la propia naturaleza de los datos orales, que contienen exclusivamente fragmentos dialogados (en los textos narrativos, sin embargo, el diálogo alterna con otras formas textuales en las que no tiene cabida el uso de la segunda persona del singular). Por otro lado, apreciamos también que la frecuencia de sabes aislado (líneas inferiores) experimenta un aumento constante en los dos corpus, mientras que no es así para las expresiones epistémicas (líneas superiores); este aumento de la frecuencia de sabes se ha intensificado en la década de los noventa.

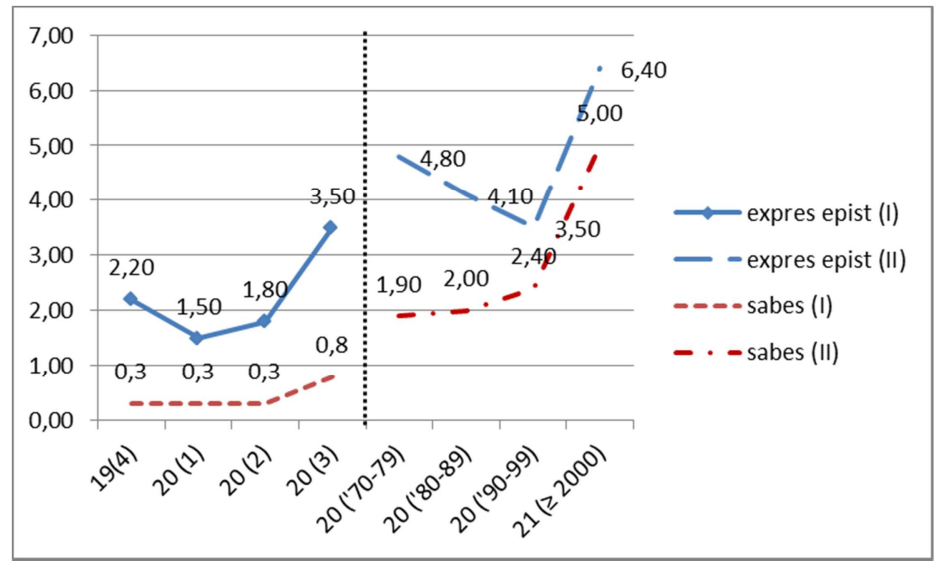

Gráfico 1. Frecuencia de expresiones epistémicas y sabes

Consideramos que los datos confirman la importancia de dos momentos clave en la evolución de sabes: (1) en la década de los 70, se impone el uso de expresiones epistémicas como "moda", seguido por la moda del sabes (Molina Martos 2005); el uso de sabes parece extenderse cada vez más, aunque no parece haber sido el caso de las expresiones epistémicas en general; y (2) en la década de los 90 y en el nuevo milenio se produce una nueva ola de uso de expresiones epistémicas, seguida por un uso creciente de sabes aislado.

Esta tendencia de uso creciente de la partícula sabes se hace aún más clara si calculamos su frecuencia relativa respecto a las demás expresiones epistémicas, reflejada en el gráfico 2.

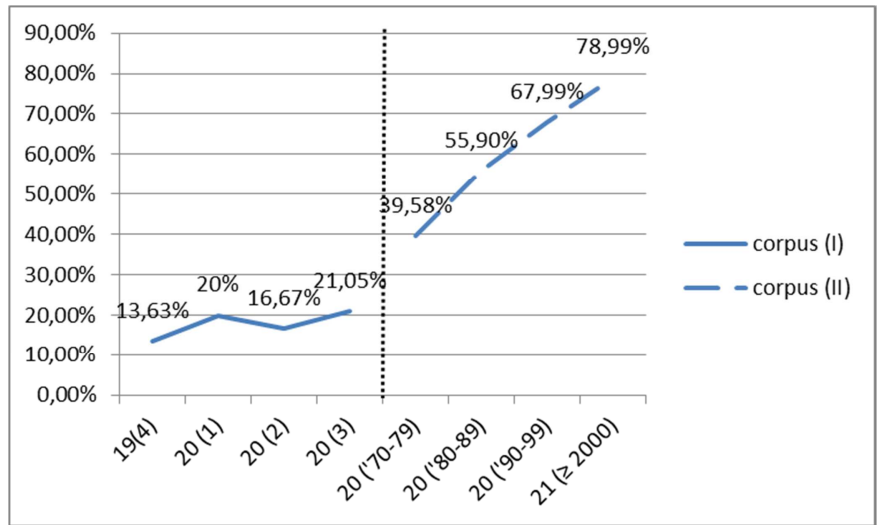


A la izquierda de la línea de puntos, se proyectan los datos del CORPUS I; a la derecha, la curva de datos del CORPUS II. Puede apreciarse que, en la época anterior a los años 70, la frecuencia relativa de sabes respecto a otras expresiones epistémicas era bastante constante, entre un $10 \%$ y un $20 \%$ (la variación puede depender del estilo de los autores). La elevación de las frecuencias empieza en el último período (20-3). Probablemente interfiere la naturaleza de los datos, de modo que en la década de los 70, ya con datos orales, las frecuencias empiezan en un nivel bastante más alto. ${ }^{9}$ Respecto a la evolución en las últimas décadas, la proporción de casos del marcador sabes sobre el total de expresiones epistémicas crece de un $40 \%$ en la década de los 70 hasta casi el $80 \%$ en la época actual. Esta tendencia sugiere, pues, una variación más amplia de expresiones epistémicas antes de 1990 y una tendencia al uso más rutinizado de sabes en contextos similares en español actual, lo cual confirma el primer parámetro de gramaticalización analizado.

\section{Correlatos morfosintácticos}

\subsection{Fijación formal y empobrecimiento sintáctico}

En segundo lugar, resulta interesante analizar la evolución en la complejidad formal de las expresiones epistémicas utilizadas, para comprobar si existe fijación formal y empobrecimiento sintáctico. A fin de averiguar si puede observarse una evolución formal en el uso de las expresiones epistémicas, se clasifican según su grado de complejidad sintáctica, y más concretamente la presencia de posibles complementos y el impacto que tiene el elemento o los elementos presente(s) en la estructura argumental. Destacamos cuatro categorías, como se recoge en la tabla 4:

(a) complejidad cero: ningún complemento o modificador presente (sabes independiente); ${ }^{10}$

(b) complejidad sintáctica baja: presencia de los modalizadores como, no o ya;

(c) complejidad sintáctica media: presencia de un sujeto (tú), de un OD nominal/pronominal, con o sin otro modalizador;

(d) complejidad sintáctica alta: presencia de una subordinada completiva como OD, con o sin sujeto expreso, con o sin modalizador.

\begin{tabular}{|c|l|}
\hline $\begin{array}{c}\text { grado } \\
\text { complejidad } \\
\text { formal }\end{array}$ & \multicolumn{1}{c|}{ expresiones epistémicas } \\
\hline cero & sabes \\
bajo & $\begin{array}{l}\text { como sabes, no sabes, ya sabes } \\
\text { sabes tú, tú sabes, como tú sabes, tú no sabes, ya sabe usted, ya sabes } \\
\text { tú, ya tú sabes, lo sabes, sabes (una) cosa, sabes qué, no lo sabes, ya lo } \\
\text { sabes, lo sabes tú, tú lo sabes, tú ya sabes algo }\end{array}$ \\
\hline
\end{tabular}

\footnotetext{
${ }^{9}$ Para establecer la comparabilidad de los subcorpus de diferente naturaleza, sería interesante extraer datos de un corpus escrito del mismo período que el corpus oral del segundo período (1975-2004); sin embargo, dado que el análisis de la comparabilidad de los datos no es el objetivo principal de este estudio, lo dejamos para un trabajo futuro.

${ }^{10}$ En el grado de complejidad que llamamos "cero", es difícil a veces distinguir la modalidad oracional (asertiva vs. interrogativa), pues los corpus tienen diferentes sistemas de transcripción y la transcripción no siempre indica la prosodia interrogativa; hemos clasificado como interrogativos algunos casos claros, a pesar de no estar transcritos con los signos de puntuación correspondientes.
} 
alto

sabes + oración, como sabes + oración, ya sabes + oración, no sabes + oración, sabes tú + oración, tú sabes + oración, no sabes tú + oración, tú no sabes + oración, tú ya sabes + oración, ya sabe usted + oración, ya sabes tú + oración, ya tú sabes + oración

Tabla 4. Clasificación de expresiones epistémicas: grado de complejidad formal

En los siguientes ejemplos se reflejan los distintos grados de complejidad formal: complejidad cero (5a-b), baja (6a-b), media (7a-b) y alta (8a-b):

(5) a. Eso dice, pero no lo creo. ¿Sabes? es como ellos. Igual. Igual que ellos. (Matute, Primera memoria, 1959)

b. ENR: hhh // ¿sabes? porque no [/] no entiende //y es complicado incluso / hacérselo entender / porque como no / sabes / a qué referirte / para lo suyo / es decir / [...]. (C-Oral-Rom)

(6) a. ¡El día que lleguen por tierra los delegados de Cantabrialta..., se prepara una buena! ¿no sabes?

- ¿Mucha fiesta?

- Los han de esperar con coches... (Pardo Bazán, La Tribuna, 1883)

b. El bien de la humanidad. Eso muy bonito eso, eso me enternece, me Somos mecenas, mecenas que son doce, ya sabes. Y naturalmente. Me interesaría también saber qué estúpido es mi compañero. (CREAOral, 1984)

(7) a. - ¿Qué le pasa a usted, señorita?

-Nada, el niño que está malito, ya sabes tú.

Petrita sonríe con cariño.

-No, el niño no tiene nada. A la señorita le pasa algo peor. (Cela, La colmena, 1951 - 1969)

b. Mira tú, Alicia, lo sabes, o sea, porque hemos estado en el mismo grupo de amigas, ya sabes tú el mismo el rollo que llevamos. (CREA-Oral, 1996)

(8) a. Más que estar conmigo te gusta el palique. ¿Sabes lo que te digo? Que si me duermo, te tienes que estar aquí. (Galdós, Fortunata, 1885 - 1887)

b. ABU: bueno / pues no //quedamos a la hora que tú / vienes / pero vente pronto //si hubiera alguna novedad /

*BAR: sí //

*ABU: / yo sabes lo que había pensado porque ése no sé lo que hará si come aquí /

*BAR: hhh // (C-Oral-Rom, 1998)

Los datos analizados, reflejados en el gráfico 3, muestran cómo desciende progresivamente el uso de expresiones epistémicas de alta complejidad sintáctica. Sin embargo, en este parámetro de la complejidad formal, más que en ningún otro, influye mucho el hecho de que el discurso sea planificado (caso del diálogo en una obra literaria) o espontáneo (caso de la conversación o la entrevista semidirigida), y por esta razón hemos preferido mostrar los resultados de los dos corpus por separado. En efecto, consideramos que, aunque era muy tentadora la idea de representar gráficamente la evolución diacrónica en la complejidad formal, los resultados no pueden tomarse como una simple línea evolutiva, por honestidad metodológica. 


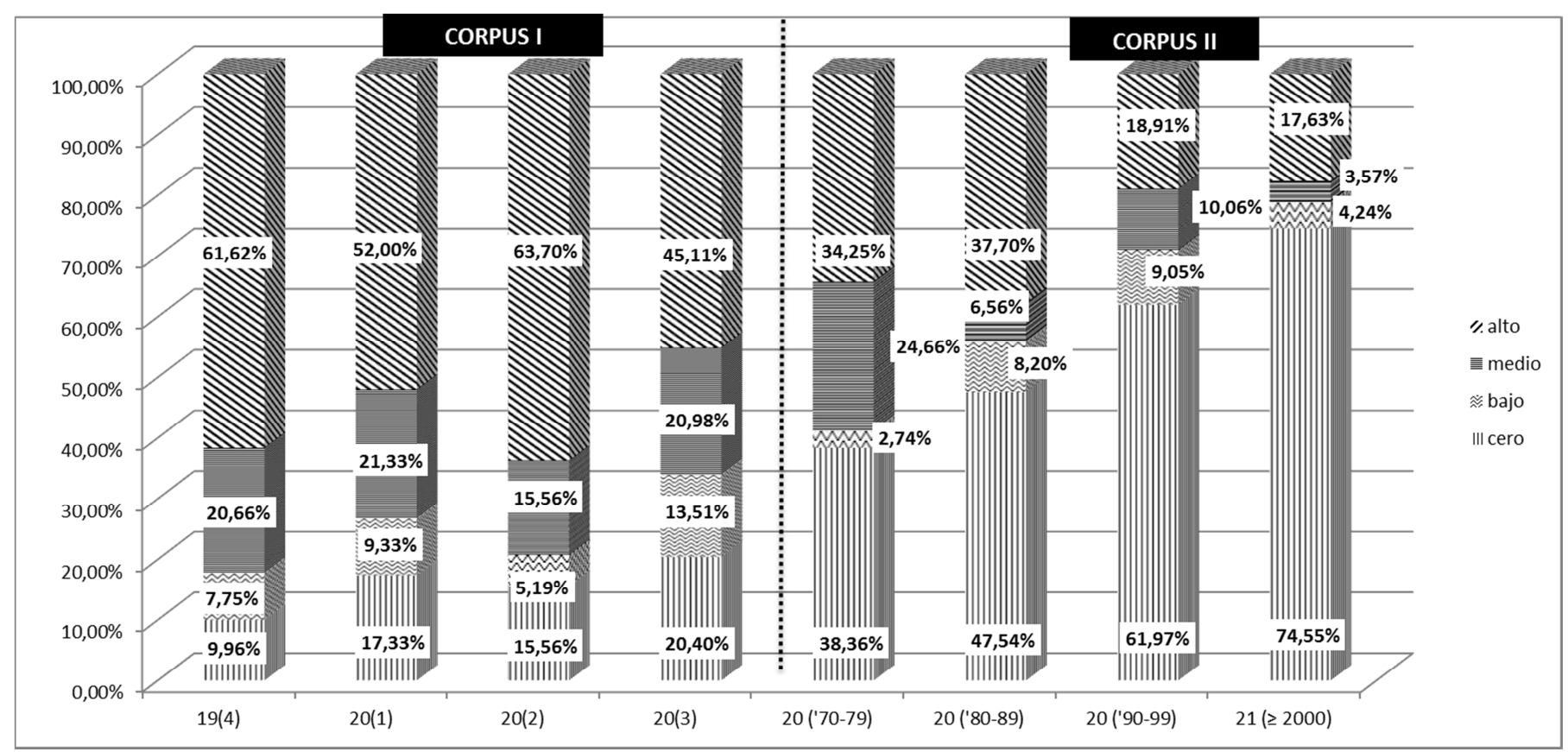

Gráfico 3. Evolución de la complejidad formal de las expresiones epistémicas

Ya en el CORPUS I se puede apreciar una tendencia constante al aumento de expresiones de complejidad cero (sabes aislado, representado en la parte inferior de cada columna), así como un descenso progresivo en el uso de las expresiones de complejidad más alta (representadas en la parte más alta). ${ }^{11}$

En términos generales, en el CORPUS II los datos apuntan a un paulatino empobrecimiento sintáctico o incluso una cancelación sintáctica en las expresiones epistémicas, con un aumento muy significativo y constante de la frecuencia de uso de sabes aislado (complejidad cero). La presencia de modalizadores junto a sabes (complejidad baja), poco significativa en la década de los 70 , aumenta entre los años 80 y 90, pero vuelve a caer a partir del año 2000. Por otro lado, disminuye a partir de los años 80 la frecuencia de sabes acompañado de sujeto o de OD nominal (complejidad media), a pesar del ligero repunte que parecen mostrar estas expresiones en la década de los 90. Respecto a las expresiones de complejidad alta, se observa que disminuyen en la década de los 90 , pero se mantiene su frecuencia después del año 2000, quizá por la polarización del valor epistémico frente a otros valores más pragmáticos (cf. infra, sección 5).

\subsection{Ampliación del alcance y autonomía de la predicación}

Por lo que se refiere a la evolución en la ampliación del alcance y la autonomía de la predicación, se acepta por lo general que los elementos "pragmaticalizados / gramaticalizados" tienden a moverse hacia posiciones periféricas, de manera que su significado incide sobre la oración entera (Adamson 2000; Company 2004, 2006 entre otros). Por tanto, parece interesante averiguar la evolución de la posición de sabes (independiente) en los corpus, para ver si confirma esta tendencia.

En la literatura sobre el discurso oral se destacan varias teorías de las posiciones diferentes que pueden ocupar las unidades lingüísticas en el discurso oral, teniendo en cuenta diferentes unidades conversacionales posibles. Sin entrar en detalle, particularmente válido es el modelo del grupo VAL.ES.CO (Teoría de las unidades conversacionales, Briz y Bordería 2010), del que retomamos dos niveles de análisis diferentes para el análisis de sabes. En primer lugar, conviene estudiar la

\footnotetext{
${ }^{11}$ Solo el segundo cuarto del siglo XX parece reflejar otro comportamiento, pues respecto al primer cuarto crece el uso de expresiones complejas y hay un ligero descenso del sabes aislado; quizá en este caso la microdiacronía debería comprender toda la primera mitad del siglo XX.
} 
posición en la intervención: sabes se define ante todo como marcador con función de "controlador de contacto", es decir, tiene una función interpersonal que se relaciona con la relación hablanteoyente. Por tanto, tiene sentido estudiar su posición en la intervención y la organización de los turnos entre los hablantes, para averiguar si la posición final en la intervención es la posición prototípica y si la posición independiente tiene función epistémica. Los niveles destacados en el análisis son: inicio, medio, final, independiente.

En segundo lugar, cuando el elemento se encuentra en posición media en la intervención, es interesante estudiar su posición respecto al enunciado o los constituyentes oracionales. De hecho, en el caso de que sabes se encuentre en posición media de la intervención, conviene refinar la clasificación, tomando como base su comportamiento frente a la sintaxis de los enunciados incluidos en una intervención (niveles de análisis posibles: entre enunciados, inicio del enunciado o subordinada, final del enunciado, interrupción, vacilación, en medio del sintagma nominal o del sintagma verbal, en medio de un complemento circunstancial). Esto deberá ser objeto de un estudio más detallado.

En el gráfico 4 se observa claramente que, desde sus primeros usos, sabes es un marcador que puede ocupar posiciones muy diversas en el discurso. Llama la atención que en todas las décadas y períodos estudiados, domina la posición en medio del turno de habla. A pesar de que los datos son de corpus de naturaleza diferente, los usos pueden considerarse bastante similares, con la salvedad de que en el CORPUS I no se documenta sabes en turno de palabra independiente.

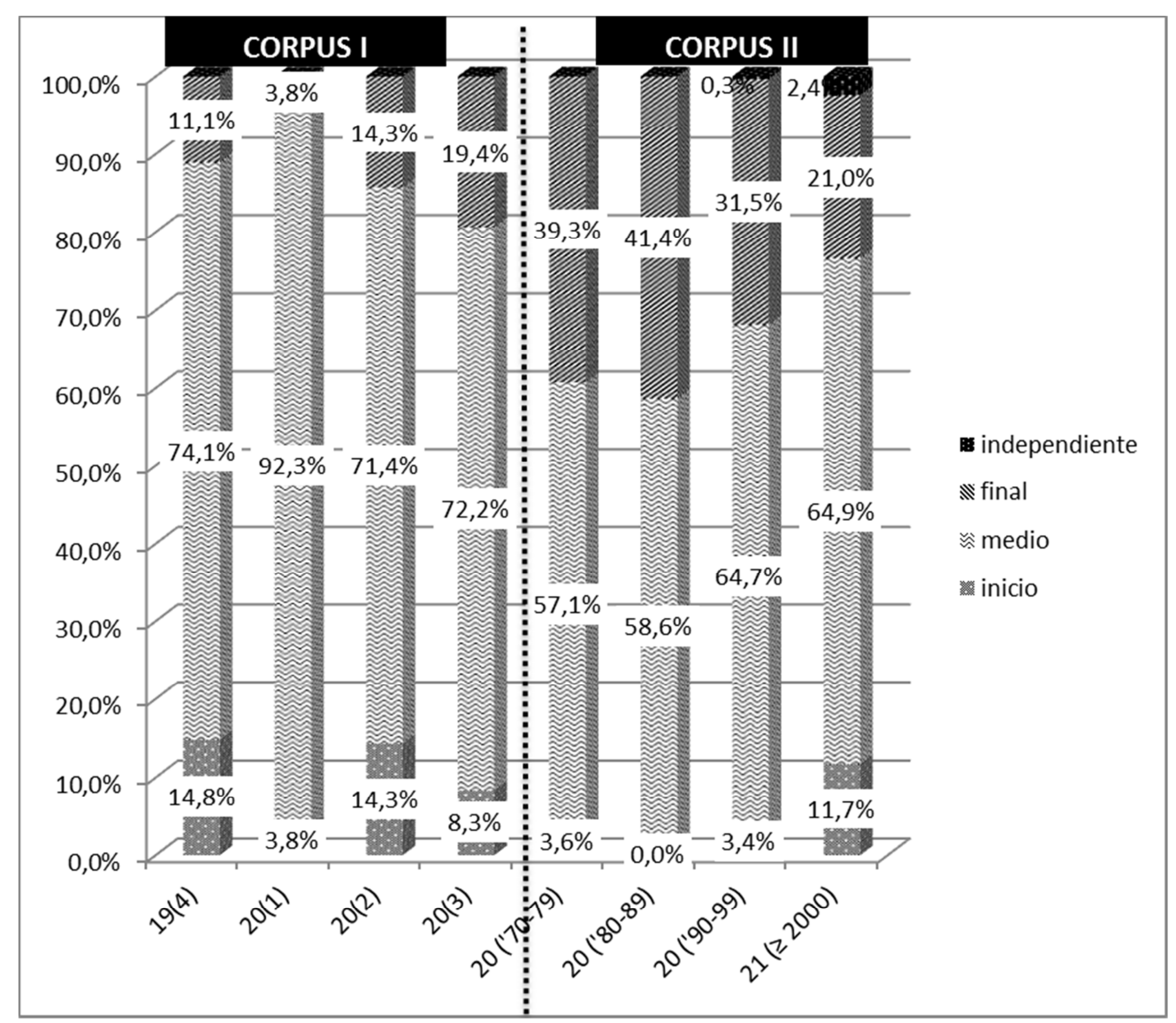

Gráfico 4. Evolución de la posición en la intervención

En el CORPUS II, a partir de la década de los 70, cuando empieza la moda del uso de sabes (Molina Martos 2005), se ve que este funciona frecuentemente como marcador con valor interpersonal de control de contacto, de acuerdo con su origen (por el valor apelativo de la segunda persona); con ese valor desarrolla fuertemente su posición final $(39.3 \%)$, posición que disminuye considerablemente si comparamos su frecuencia a partir del año 2000 (21\%). 
(9) <H1> Pues no sé que va a pasar. Al final yo creo que... los hijos no van a querer separarse, ¿sabes?

$<\mathrm{H} 2>$ Claro. (Corlec, 1991)

Observamos entonces, ya en el siglo XXI, un aumento de la posición intermedia y de la posición inicial, paralelo al desarrollo de su función fática, con la intención de llamar la atención del interlocutor (ver infra, sección 5) (10a-b). En los casos de posición intermedia, sabes puede ser también apelativo (11a) o muletilla ${ }^{12}$ (11b). El uso independiente no se documenta en los corpus hasta los 90 y aumenta ligeramente en el nuevo milenio; muchas veces es el verdadero valor epistémico (12).

(10) a. [...] trabábase entre los dos sabroso coloquio de chismografía burocrática. - ¿Sabes...?-decía Pantoja-. Hoy salieron calentitos dos oficiales primeros y un jefe de Administración. (Galdós, Miau, 1888)

b. *INM: no sé / yo creo que también la cosa es tener suerte //

*PAT: hhh //sí / <porque xxx>

*INM: ¿Sabes? porque a lo mejor [...]. (C-Oral-Rom, 2001)

(11) a. Oye, me estoy poniendo un poco nerviosa, Zacarías. Pero me gusta estar contigo, ¿sabes? Digo yo si será por eso mismo, a lo mejor. (Sánchez Ferlosio, El Jarama, 1956)

b. *ENR: <bueno> te te sigo que sabes mira / hay un grupo / de primero // un solo grupo // con quince alumnos //

*INM: y /

*ENR: es decir <que>

*INM: / <hay plazas> para ... más o menos //

(12) I: ¿sabes? o sea que no sé qué qué tipo de concienciación ten tendremos las personas

E: sí

I: ¿Sabes?

E: hombre como hay que (PRESEEA, 2001)

Basándonos en los datos, podemos concluir que desde finales del siglo XIX hasta principios del siglo XXI se observa un movimiento triple: (1) la gama de expresiones epistémicas con base léxica en el verbo sabes se restringe gradualmente; (2) sabes continúa su propio proceso de gramaticalización, lo cual se refleja en una gama más amplia de las posiciones que puede ocupar (movilidad sintáctica); y (3) al mismo tiempo se mantiene como expresión con valor epistémico pleno.

\section{Blanqueamiento semántico: funciones y progresiva pragmaticalización}

El último apartado analiza si se observa en los corpus alguna evolución en las funciones que puede cumplir el elemento sabes El objetivo no es tratar de clasificar los ejemplos según la proliferación de valores diferentes que puede cumplir el marcador. Al contrario, estamos de acuerdo con House (2009: 189) cuando dice: [...] despite the many different functions discourse makers like you know can assume in real talk, any unified account is more helpful than the assuming a host of different functions and uses" [a pesar de las muchas funciones diferentes que pueden asumir marcadores discursivos como you know en el habla real, un acercamiento unificado es más útil que asumir una multitud de funciones y usos diferentes]. Por lo tanto, para el estudio de corpus se destacan tres macrofunciones (intersubjetiva, subjetiva y muletilla), sin olvidar que estas funciones pueden solaparse; creemos que, aunque siempre existe una semántica intersubjetiva subyacente por su forma, sabes puede estar más o menos blanqueado.

\footnotetext{
${ }^{12}$ Siguiendo a Cortés Rodríguez (1991), la noción de muletilla se define como un elemento lingüístico de relleno que da tiempo al hablante para organizar su discurso. Entonces gradualmente se va desarrollando una gama más amplia de posiciones en el ámbito medio de la intervención. Un estudio piloto muestra que de acuerdo con su origen, la posición prototípica, también en medio de una intervención, es la posición final de enunciado, aunque se desarrollan progresivamente otras posiciones, sobre todo con funciones de interrupción y vacilación.
} 
Para limitar al máximo los efectos de una clasificación intuitiva y subjetiva de los datos, se deduce la función semántico-pragmática del marcador con base en dos parámetros: (1) la semántica del contenido del mensaje y (2) la presencia de ciertos indicios léxicos / elementos léxico-formales en el contexto. La tabla 5 muestra esos indicios contextuales que se asocian a cada macrofunción semántico-pragmática.

\begin{tabular}{|l|l|l|}
\hline \multicolumn{1}{|c|}{ macrofunción } & \multicolumn{1}{|c|}{ significado pragmático } & \multicolumn{1}{c|}{ indicios contextuales } \\
\hline $\begin{array}{l}\text { intersubjetiva } \\
\text { (orientación }\end{array}$ & $\begin{array}{l}\text { “Compartes este conocimiento } \\
\text { conmigo?” "me entiendes”; } \\
\text { interlocutor) }\end{array}$ & $\begin{array}{l}\text { respuestas (sí, no, claro, ya...), } \\
\text { uso de imperativo dirigido al } \\
\text { interlocutor }\end{array}$ \\
$\begin{array}{l}\text { sujetiva } \\
\text { (orientación } \\
\text { hablante) }\end{array}$ & $\begin{array}{l}\text { hablante enfatiza parte del } \\
\text { mensaje, hablante comunica } \\
\text { nuevas informaciones, emociones; }\end{array}$ & $\begin{array}{l}\text { exclamación, referencia al } \\
\text { "yo”, tono negativo/positivo, } \\
\text { uso superlativos, prefijos } \\
\text { salor expresivo }\end{array}$ \\
$\begin{array}{l}\text { muletilla } \\
\text { (orientación } \\
\text { discurso) }\end{array}$ & $\begin{array}{l}\text { enfoque en contenido mensaje, } \\
\text { flujo de ideas }\end{array}$ & $\begin{array}{l}\text { repeticiones, puntos } \\
\text { suspensivos, pausas, palabras } \\
\text { vagas (por ejemplo cosa) }\end{array}$ \\
\hline
\end{tabular}

Tabla 5. Valores semántico-pragmáticos e indicios contextuales

Así, en el corpus se documentan ejemplos de valor intersubjetivo, orientado hacia el interlocutor, más próximo al valor léxico pleno de sabes (13a-b); casos de valor subjetivo, dirigido al hablante (14a-b) y, por último, usos más discursivos, como muletilla (15a-b):

(13) a. De este embarazo no le digas nada a mamá todavía, ¿sabes?, para qué se va a andar preocupando. (Martín Gaite, Entre visillos, 1958)

b. *VER: que al final / voy a hacer / las dos / o sea / creo que el orden / no ha sido el bueno / ¿sabes?

*BEA: ya // [...]. (C-Oral-Rom, 2001)

(14) a. ¡Es tan prosaico! Es que yo no puedo casarme, ¿sabes? No sirvo para tal vida: (Pardo Bazán, La Quimera, 1905)

b. Yo al principio de retirarme creí que ya sin hacer nada me iba a morir, ¿sabes? (CORLEC, 1991)

(15) a. Es que yo le he contado ¿sabes?, que siempre me has ayudado a aprobar (Martín Gaite, Entre visillos, 1958)

b. a mí eso a mí nunca se me<alargamiento/> / se me ocurriría ¿no? // y sin embargo ahora pues sí que es // es es / ¿sabes? / cuando vas por algunas zonas pues / tienes más<alargamiento/> / no sé o sea no vas (PRESEEA, 2001)

Sin embargo, cabe destacar que la clasificación se resiste en muchos casos, como podemos apreciar en los ejemplos (16a-b). La plurifuncionalidad o ambigüedad entre el valor intersubjetivo y la simple muletilla es patente en (16a), mientras que en (16b) la ambiguiedad funciona entre el valor intersubjetivo y el subjetivo:

(16) a. Mira, tráeme agua azucarada... templadita, ¿sabes? Tengo sed. (Galdós, Fortunata, 1885-1887)

b. Aquí estoy, no llores... Sólo iba al bar ¿sabes? ¿Y quieres saber a qué? Pues a jugar un rato, (Marsé, Últimas tardes con Teresa, 1966)

Podemos afirmar que sabes casi nunca se pierde el sentido original, caracterizado por la interacción (impuesta por la persona verbal) y por la referencia epistémica que caracteriza al lexema.

Conviene ahora examinar la evolución en los valores semántico-pragmáticos del elemento sabes, es decir, analizar si se observa o no una tendencia a usos más blanqueados. De acuerdo con el contenido léxico originario de sabes y su forma gramatical (verbo epistémico y verbo en 2. ${ }^{a}$ persona del singular), el progreso en el blanqueamiento sería el siguiente:

valor intersubjetivo > valor subjetivo > uso como muletilla 
Como se puede apreciar en el gráfico 5, en el CORPUS I domina claramente el uso intersubjetivo: en cualquiera de los períodos que abarca el subcorpus, casi la mitad de los casos tiene valor intersubjetivo (parte inferior de las columnas), ya sea epistémico u otro. En estos casos, de acuerdo a su forma, sirve para facilitar o reforzar la implicación del interlocutor en el acto de comunicación, ya sea aludiendo a un conocimiento compartido, ya sea con un valor más apelativo. Sin embargo, llama la atención la alta frecuencia de valor expresivo (subjetivo) de sabes, ya en este período (parte intermedia de las columnas), y también algunos casos de uso como muletilla antes de 1975. De cualquier forma, el bajo número de ejemplos de sabes independiente interrogativo en el CORPUS I obliga a observar con cautela los datos estadísticos, por el peso que uno solo de los ejemplos tiene sobre el conjunto. ${ }^{13}$

Por lo que se refiere al CORPUS II, donde las estadísticas son más fiables por responder a un número mayor de ejemplos, se observa un desarrollo cuantitativo progresivo de la función expresiva subjetiva, por lo menos en la década de los 70 . Una vez pasada esa "moda", en los 80, adquiere otra vez más valor intersubjetivo, aunque a partir de los 90 y sobre todo en el siglo XXI parece dominar cada más su función de muletilla. De nuevo, estos datos parecen sugerir la existencia dos tipos de sabes: el de los años 70, que sería un sabes subjetivo (expresivo), y el de los 90 y 2000, que sería un sabes expresivo pero sobre todo blanqueado hasta convertirse en muletilla (función discursiva). Es muy probable que la influencia del inglés you know haya pesado sobre este sabes español, hasta el punto de hacerle perder su prototípica entonación interrogativa. ${ }^{14}$

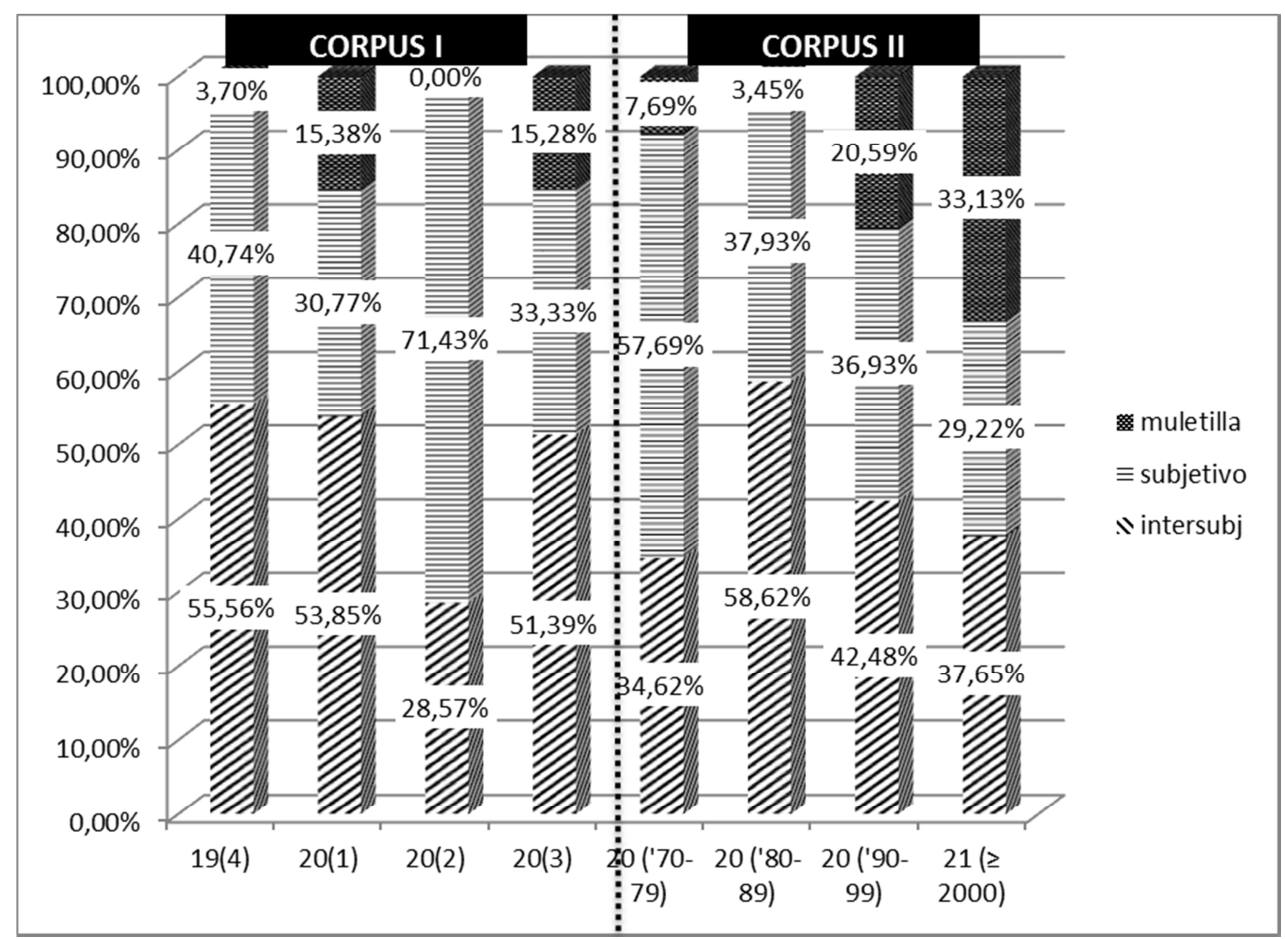

Gráfico 5. Evolución de los valores semántico-pragmáticos.

\section{Conclusiones}

Una vez analizados los datos, se puede dar respuesta a las dos preguntas de investigación planteadas inicialmente, que repetimos aquí por motivos de claridad:

\footnotetext{
${ }^{13}$ A modo de ejemplo, un solo caso de sabes muletilla en el siglo 19-4 representa el 3,5\% de los usos.

${ }^{14}$ Como ya hemos señalado, el establecimiento de la modalidad interrogativa o enunciativa a veces plantea problemas. Precisamente la determinación de su valor semántico-pragmático nos ha llevado a incluir como casos de marcador algunos ejemplos en los que no había marcación expresa de la modalidad interrogativa a través de los signos de puntuación (casos en que podía estar neutralizada o al menos no haber sido reflejada convenientemente por el autor de un texto literario o por el responsable de la transcripción de un texto oral).
} 
(i) ¿Cómo se describe empíricamente la evolución del uso de las expresiones epistémicas observando ejemplos consecutivos del español moderno y contemporáneo?, ¿cuál es la frecuencia de sabes y sus variantes (ya sabes, no sabes, tú sabes, etc.) en el corpus?

(ii) ¿En qué medida esta evolución confirma el estatus gramaticalizado de sabes?, ¿en qué medida sabes cumple entonces las características de los marcadores del discurso?

Respecto a la primera, los datos demuestran que no se puede estudiar el marcador sabes de manera aislada, sin integrarlo en la red más amplia de expresiones epistémicas que tiene a su disposición el hispanohablante. Hemos analizado la frecuencia relativa de sabes y sus variantes en el corpus, confirmando la idea de que la evolución de este marcador tiene su origen en expresiones epistémicas más complejas con el mismo núcleo léxico, que presentan desde el siglo XIX los mismos valores que sabes (ya sabes, no sabes, tú sabes, sabes lo que + oración, etc.). Por otro lado, sabes (independiente y con entonación interrogativa), con distintos valores discursivos, se documenta ya al menos desde finales del siglo XIX. Por tanto, no es una creación reciente.

Por lo que se refiere a la segunda pregunta de investigación, a saber, la naturaleza de sabes, ha quedado patente que su comportamiento confirma su estatus de marcador de discurso, al menos desde fines del siglo XX. Se cumplen los criterios asociados con la gramaticalización, si bien en distinta medida: (a) aumento de frecuencia de uso, tanto absoluta (sobre el total de palabras) como relativa (sobre el conjunto de expresiones epistémicas con el mismo núcleo léxico); (b) fijación formal y reducción de la complejidad sintáctica, hasta llegar a la descategorización y la cancelación de la sintaxis propia de la forma original (en términos de Company 2004); (c) ampliación de alcance y autonomía respecto a la predicación: el cambio de ámbito se refleja en la variación de posición, así como en la movilidad (tanto en el enunciado como en la intervención); y (d) por último, blanqueamiento: pérdida de contenido semántico y progresivo aumento de usos más alejados de lo intersubjetivo. La pragmaticalización es patente por el aumento de su uso como muletilla, cuando se produce un blanqueamiento casi total y se introduce un funcionamiento con valor solo discursivo.

Además, tanto el análisis de su comportamiento formal en el discurso como el de sus valores pragmático-semánticos apuntan a dos "modas" diferentes en el uso del sabes: una en la década de los 70, cuando el marcador sabes desarrolla fuertemente su función (inter)personal y ocupa a menudo la posición final, y una segunda moda de sabes a finales de los años 90 principios de los años 2000, que parece ser diferente y coincide con un uso más frecuente de la posición inicial e intermedia. Así, al menos en las décadas más recientes, parece desplazarse de su función prototípica interpersonal en la periferia derecha a desarrollar otras funciones en la periferia izquierda, un desplazamiento que es común a otros marcadores del discurso que sufren procesos de subjetivización o intersubjetivización (Beeching y Detges 2014). Postulamos que la segunda ola es influencia del inglés you know, un hecho que puede estar relacionado con la presencia cada vez mayor del inglés en la comunidad hispanohablante y con el mayor grado de conocimiento de la lengua inglesa entre los jóvenes (por ejemplo, Medina López 1996; Pountain 1999), al menos en la Península, que es de donde proceden los datos.

Respecto al objetivo metodológico de este artículo, hemos llegado a la conclusión de que, para los estudios diacrónicos sobre marcadores, no son comparables totalmente los datos extraídos de un corpus literario y los extraídos de corpus orales, como era previsible. La diferencia en la planificación, por ejemplo, puede afectar en gran medida a los resultados de frecuencia relativa del marcador y de las expresiones epistémicas en general, así como al parámetro "complejidad formal". Sin embargo, otros parámetros como el valor semántico-pragmático pueden no resultar tan afectados por la naturaleza del corpus. Es cierto que existe una necesidad real de acudir a textos literarios para documentar el registro oral de épocas pasadas para las que faltan corpus orales, pero se debe atender, por exigencia metodológica, a la necesidad de relativizar los resultados y no presentarlos como un continuum diacrónico. Esto es lo que ha motivado la presentación de microanálisis independientes para los dos subcorpus, separados también en los gráficos. Para 
ampliar el trabajo, es necesario incluir textos literarios correspondientes al mismo período cronológico que abarca el CORPUS II de textos orales, es decir, contar con datos de obras literarias escritas entre 1976 y la actualidad, lo cual nos permitiría conocer el grado de comparabilidad entre los dos subcorpus manejados.

Por último, queremos destacar que este trabajo forma parte de una investigación más amplia que dará cabida a algunas consideraciones que, por motivos evidentes, no hemos podido incluir aquí, como el análisis de la influencia en los resultados de los diferentes estilos individuales (el del autor del tex to literario o el idiolecto particular de un informante) o el análisis de la influencia de factores extralingüísticos (sexo del hablante, nivel sociocultural, etc.) para explicar la evolución del marcador y, sobre todo, su difusión.

\section{Fuentes}

Cresti, Emanuela; Moneglia, Massimo (2005): C-ORAL-ROM. Integrated Reference Corpora for Spoken Romance Languages. Amsterdam: Benjamins.

Esgueva, Manuel; Cantarero Margarita, (eds.) (1981): El habla de la ciudad de Madrid. Materiales para su estudio, Madrid: Consejo Superior de Investigaciones Científicas.

Fernández-Ordóñez, Inés: Corpus Oral y Sonoro del Español Rural (COSER) [en línea: http://www.uam.es/coser].

Marcos Marín, Francisco A.: Corpus Oral de Referencia del Español Contemporáneo (CORLEC) [en línea: http://www.lllf.uam.es/ESP/Corlec.html].

Moreno Fernández, Francisco et al.: Proyecto para el Estudio Sociolingüístico del Español de España y de América (PRESEEA) [en línea: http://preseea.linguas.net/].

Real Academia Española (RAE): Banco de datos (CREA) (en línea). Corpus de referencia del español actual [en línea: http://www.rae.es].

Real Academia Española (RAE): Banco de datos (CORDE) (en línea). Corpus Diacrónico del Español [en línea: http://www.rae.es].

\section{Referencias bibliográficas}

Adamson, Sylvia (2000): "A lovely little example: Word order options and category shift in the premodifying string", en: Fischer, Olga; Rosenbach, Anette; Stein, Dieter (eds.): Pathways of Change: Grammaticalization in English, Amsterdam / Philadelphia: John Benjamins, pp. 39-66.

Beeching, Kate; Detges, Ulrich (2014): "Introduction", en: Beeching, Kate; Detges, Ulrich (eds.): Discourse Functions at the Left and Right Periphery Crosslinguistic Investigations of Language Use and Language Change, Leiden: Brill, pp. 1-23.

Boyero Rodríguez, María José (2002): Los marcadores conversacionales que intervienen en el desarrollo del diálogo, Salamanca: Publicaciones Universidad Pontificia.

Briz Gómez, Antonio; Pons Bordería, Salvador (2010): "Unidades, marcadores discursivos y posición”, en: Loureda, Óscar; Acín, Esperanza (eds.): Los estudios de marcadores del discurso en español, hoy, Madrid: Arco/Libros, pp. 327-358.

Bybee, Joan (2006): "From usage to grammar: the mind's response to repetition", Language 82/4, pp. 711-733.

Company, Concepción (2004): "Gramaticalización por subjetivización como prescindibilidad de la sintaxis", Nueva Revista de Filología Hispánica 52 /1, pp. 1-28.

- (2006): "Zero in syntax, ten in pragmatics", en: Athanasiodou, Angeliki ; Canakis, Costas; Cornillie, Bert (eds.): Subjectification: various paths to subjectivity, Berlin/New York: Mouton de Gruyter, pp. 375-398. 
Cortés Rodríguez, Luis (1999): Sobre conectores, expletivos y muletillas en el español hablado, Málaga: Editorial Librería Ágora.

Cuenca, Maria Josep (2013): "The fuzzy boundaries between discourse marking and modal marking", en: Liesbeth Degand, Bert Cornillie, Paola Pietrandrea (eds.): Discourse markers and modal particles. Categorization and description, Amsterdam: John Benjamins, pp. 191216.

Cuenca, Maria Josep; Marín, Maria Josep (2000): "Verbos de percepción gramaticalizados como conectores. Análisis contrastivo español-catalán”, en: Maldonado, Ricardo (ed.): Estudios cognoscitivos del español, monográfico de la Revista Española de Lingüística Aplicada, pp. 215-238.

Dostie, Gaétane; Sève, Suzanne de (1999) : "Du savoir à la collaboration. Étude pragma-sémantique et traitement lexicographique de t'sais", Revue de Sémantique et Pragmatique 5, pp. 11-35.

Enghels, Renata; Vanderschueren, Clara; Bouzouita, Miriam (2015): "Panorama de los corpus y textos del español peninsular contemporáneo", en: Roegiest, Eugeen; Iliescu, Maria (eds): Manuel des anthologies, corpus et textes romans 7, pp. 147-170.

Fuentes Rodríguez, Catalina (2009): Diccionario de conectores y operadores del español, Madrid: Arco Libros.

Garachana, Mar (1999): “Los procesos de gramaticalización”, Moenia 5, pp. 155-172.

- (2008): "En los límites de la gramaticalización. La evolución de encima (de que) como marcador del discurso", Revista de filología española 88/1, pp. 7-36.

Hernando Cuadrado, Luis Alberto (2015): "El registro coloquial en la obra literaria", en: Hernando Cuadrado, Luis Alberto (coord.): Lengua y discurso. Madrid: Visor Libros, pp. 127-165.

Hopper, Paul J.; Traugott, Elizabeth C. (2003): Grammaticalization, Cambridge: Cambridge University Press.

House, Juliane (2009): "Subjectivity in English as Lingua Franca discourse: The case of you know", Intercultural Pragmatics 6/2, pp. 171-193.

Kluge, Bettina (2011): "Camino de un marcador del discurso: una comparación del español ¿sabes? con el francés tu sais y el inglés you know", en: Aschenberg, Heidi; Loureda, Óscar (eds.): Marcadores del discurso: de la descripción a la definición, Madrid/Frankfurt: Iberoamericana/Vervuert, pp. 305-341.

López Serena, Araceli; Borreguero Zuloaga, Margarita (2010): "Los marcadores del discurso y la variación lengua hablada vs. lengua escrita”, en: Loureda, Óscar y Acín, Esperanza (eds.): Los estudios sobre marcadores del discurso en español, hoy, Madrid: Arco Libros, pp. 415495.

Macaulay, Ronald (2002): "You know, it depends", Journal of Pragmatics 34, pp. 749-767.

Martín Zorraquino, María Antonia; Portolés Lázaro, José (1999): "Los marcadores del discurso", en: Bosque, Ignacio; Demonte, Violeta (eds.): Gramática descriptiva de la lengua española, Vol. III, Madrid: Espasa Calpe, pp. 4051-4214.

Medina López, Javier (1996): El anglicismo en el español actual, Madrid: Arco Libros.

Molina Martos, Isabel (2005): "La moda del ¿sabes? en Madrid: un análisis sociolingüístico", en: Lingüística y literatura. Homenaje a Antonio Quilis, Madrid: CSIC/UNED/Universidad de Valladolid, pp. 1025-1036.

Ortega Olivares, Jenaro (1986): “Aproximación al mecanismo de la conversación: apéndices justificativos", Verba 13, pp. 269-290.

Pons Bordería, Salvador (2014): "El siglo XX como diacronía: intuición y comprobación en el caso de o sea", RILCE 30/3, pp. 985-1016.

Pountain, Christopher J. (1999): "Spanish and English in the 21st century", Donaire 12, pp. 33-42.

Posio, Pekka (2014): "Subject expression in grammaticalizing constructions. The case of creo and acho 'I think' in Spanish and Portuguese", Journal of Pragmatics 63, pp. 5-18.

Santos Río, Luis (2003) Diccionario de partículas, Salamanca: Luso-española de ediciones. 
Tanghe, Sanne (2013): "Acerca de los intensificadores vaya y qué: un estudio comparativo", Bulletin of Hispanic Studies 90/7, pp. 751-769.

Thompson, Sandra A.; Mulac, Anthony (1991): "The discourse conditions for the use of the complementizer that in conversational English", Journal of pragmatics 15, pp. 237-251.

Van Bogaert, Julie (2011): "I think and other complement-taking mental predicates: A case of and for constructional grammaticalization", Special issue of Linguistics 42/2, pp. 295-332.

Vincent, Serge ; Darbaky, Sarah; Mettouchi, Amina (2009): "The grammaticalization of you know. From shared knowledge to control over the co-speaker", English Text Construction 2/2, pp. 209-227. 